\title{
The relevance of a fast and early diagnosis of necrotizing fasciitis in the Emergency Department: a case report
}

\author{
Marianna Gregorio, Antonio Villa \\ Emergency Department, Desio Hospital, Desio (MB), Italy
}

\begin{abstract}
We report a case of necrotizing fasciitis in an 84-year-old man affected by diabetes mellitus. The patient was admitted in the Emergency Department of our hospital because of an acute and strong left leg pain that began almost $8 \mathrm{~h}$ before admission. The left leg had an increased size and a movement limitation, with a hard hematoma in the left thigh with subcutaneous crepitus. The lesion became worse and larger rapidly, with a wide extension from the back to the popliteal fossa. An antimicrobial therapy was immediately started with morphine for pain. A surgical debridement was performed, but the patient died for multi-organ failure. Necrotizing fasciitis is a rare and mortal disease, the early diagnosis is a challenge for the Emergency Department where patients are admitted and assessed primarily.
\end{abstract}

\section{Introduction}

Necrotizing fasciitis (NF) is a severe and rare soft tissue infection that starts from the superficial fascia and can involve the underlying tissues.

It can develop from skin lesions as e.g., insect bite, needle puncture, surgical wound, abscess, injury and also areas affected by Herpes zoster or a venous ulcer.

Risk factors of NF are diabetes mellitus, immunosuppression, intravenous drug abuse, abdomen trauma, age, malnutrition, vascular diseases and obesity.

It may spread rapidly leading to significant morbidity and mortality.

The mortality is high, ranging from $20 \%$ to up to $50 \%{ }^{1,2}$

Correspondence: Marianna Gregorio, Emergency Department, Desio Hospital, via Mazzini 1, 20832 Desio (MB), Italy. Tel.: +39.0362.383021 - Fax: +39.0362.385742.

E-mail: mariannagregorio@hotmail.com

Key words: Necrotizing fasciitis; early diagnosis; emergency; diabetes mellitus.

Contributions: the authors contributed equally to this work.

Conflict of interest: the authors declare no potential conflict of interest.

Received for publication: 25 October 2015.

Revision received: 23 December 2015.

Accepted for publication: 28 December 2015.

This work is licensed under a Creative Commons Attribution NonCommercial 4.0 License (CC BY-NC 4.0).

CCopyright M. Gregorio and A. Villa, 2016

Licensee PAGEPress, Italy

Italian Journal of Medicine 2016; 10:223-225

doi:10.4081/itjm.2016.671
Early diagnosis of NF is crucial in the Emergency Department and it is often delayed because of similarities with other soft tissue diseases as cellulitis. The treatment consists of early broad-spectrum antibiotic therapy and aggressive surgical debridement.

We report a case of NF managed in the emergency department of our hospital.

\section{Case Report}

An 84-year-old man was admitted in the Emergency Department because of acute left leg pain, started almost $8 \mathrm{~h}$ before at home. At the admission, subjective dyspnea and cyanotic skin were noted by nurses.

The patient was affected by diabetes mellitus and he did not report any trauma neither laceration. He reported fever in the days before admission.

On admission, blood pressure was 160/120, cardiac rate was 120 pulses, oxygen saturation was $100 \%$ and temperature was $36^{\circ} \mathrm{C}$. He was awake with a Glasgow coma scale score 15 .

Lung auscultation was characterized by humid rumors.

The left leg had an increased size and a movement limitation because of the severe pain, with hematoma in the left thigh that was hard with subcutaneous crepitus.

Laboratory data showed leukocytosis $(17,800 /$ $\left.\mathrm{mm}^{3}\right)$, high serum creatinine kinase $(9373 \mathrm{U} / \mathrm{L})$, normal hemoglobin $(13.6 \mathrm{~g} / \mathrm{dL})$, low serum sodium (132 $\mathrm{mMol} / \mathrm{L})$, high serum procalcitonin $(77 \mathrm{ng} / \mathrm{mL})$ and serum C reactive protein $(183 \mathrm{mg} / \mathrm{L})$, high serum glucose $(223 \mathrm{mg} / \mathrm{dL})$ and high serum creatinine $(2.55$ $\mathrm{mg} / \mathrm{dL}$ ).

Peripheral blood hemocultures were taken, where Gram-positive bacteria will grow afterwards (Clostrid- 
ium subterminale and Clostridium histolyticum).

Analysis of blood gases showed a $\mathrm{pH}$ value of 7.46, $\mathrm{pO}_{2} 99 \mathrm{mmHg}, \mathrm{pCO}_{2} 14 \mathrm{mmHg}$, lactates 8 $\mathrm{mmol} / \mathrm{L}$ and $\mathrm{pO}_{2} / \mathrm{FiO}_{2}$ ratio 471 .

Chest radiography was normal.

Abdomen and left leg computed tomography showed subcutaneous gas extended from the lumbarsacral area to the popliteal fossa, leading to a diagnosis of NF (Figures 1-3).

During the stay in the Emergency Department (4 h), the patient's conditions became worse, the pain was still strong and the hematoma became larger and extended from the back to the whole left leg.

A treatment with antimicrobial therapy was started [clindamycin $900 \mathrm{mg}$ and gentamicin $80 \mathrm{mg}$ intravenously (i.v)], with morphine for pain and i.v. liquid support.

A surgical debridement was performed after $5 \mathrm{~h}$ from admission, but the patient cannot survive and died after that for multi-organ failure in Intensive Care Unit.

\section{Discussion and Conclusions}

Early diagnosis of NF is difficult, due to its nonspecific presentation and initial symptoms that are in common with other soft tissue diseases as cellulitis.

The challenge for the Emergency Department's team is to early detect and recognize NF cases in order to: i) start a correct therapy and to address patients rapidly to surgical debridement; ii) reduce mortality.

The role of the Emergency Department physician is crucial and, at the same time, very difficult. The physicians should recognize rapidly the NF signs and symptoms, assess laboratory and radiological exams and start antimicrobial therapy as soon as possible. Moreover, since the first suspect of NF, it is necessary to alert the surgical team for the debridement.

Often in the Emergency Department physicians work with a high level of stress and have to assess a high number of patients, this is another point to consider that may delay the NF diagnosis or make it wrong.

For this reason, the physicians have to consider carefully the clinical features and the laboratory data.

In literature, there are numerous studies that underline the NF signs and symptoms and the use of clinical and laboratory scores. These instruments could help physicians in the correct management of a patient with a NF suspect.

A review of Cheung et al. ${ }^{3}$ stressed the clinical difference between NF and cellulitis, in particular in NF a pain out of proportion to the physical findings is the only distinguishing feature. Moreover, a generalized erythematous rash and a toxic appearance are more likely to the NF than cellulitis.

In a more recent review Borschitz et al. ${ }^{4}$ described the clinical signs for NF: erythematous and edematous skin lesions, blister formation, ulceration, necrosis and crepitus. NF patients complained about strong pain that was not described in cellulitis patients.

Goh et al. ${ }^{5}$ made a systematic review about NF.

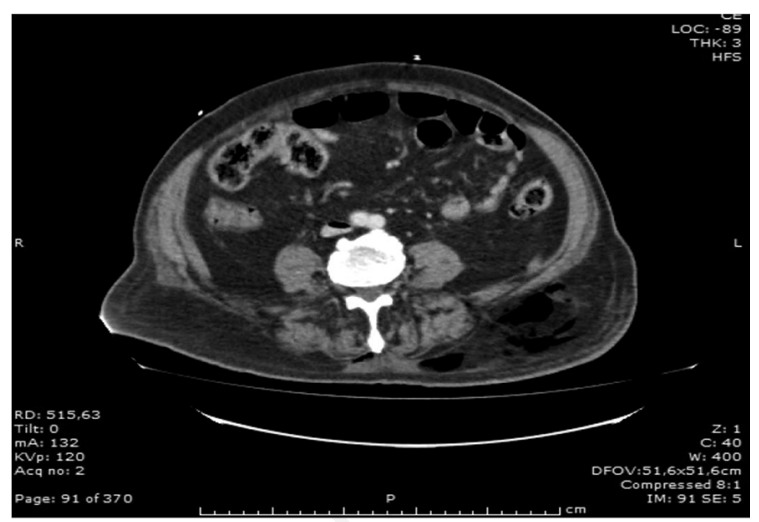

Figure 1. Gas material in lumbar-sacral area.

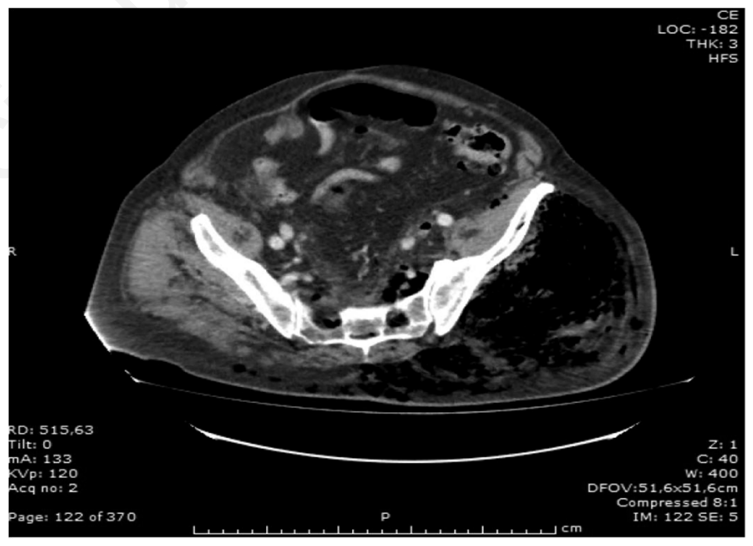

Figure 2. Gas material in left gluteus.

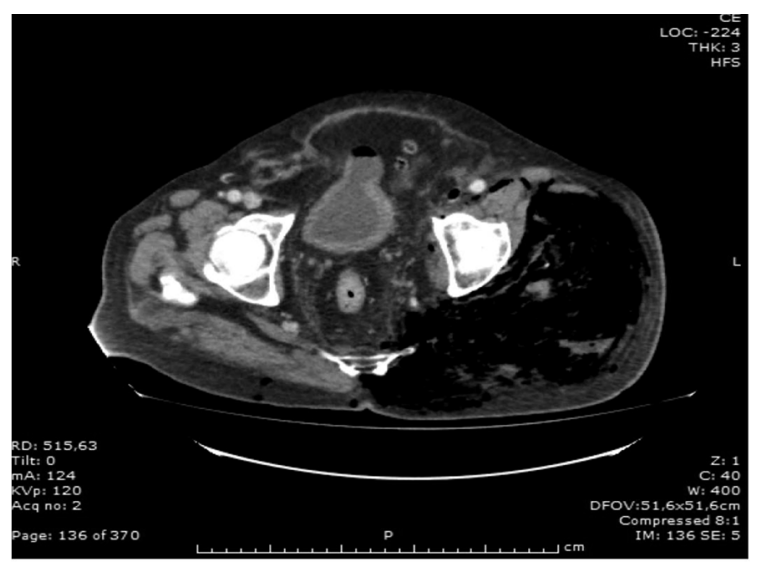

Figure 3. Gas material in left thigh. 
They consider a total amount of 1463 patients, in which the three early clinical features were: swelling $(80.8 \%)$, pain (79\%) and erythema (70.7\%) that are non-specific for NF, causing a misdiagnosis in threequarters of patients. To early detect NF cases the authors stress the use of the finger test that consists to make a $2 \mathrm{~cm}$ incision in the suspected area once anesthesia has been applied. The test is positive for NF if the index finger can dissect the subcutaneous tissue from the deep fascia.

Concerning laboratory data, a score was created to stratify patients and identify NF cases. This score is called LRINEC (laboratory risk indicator for necrotizing fasciitis) and it uses six parameters: ${ }^{6}$ i) $\mathrm{C}$ reactive protein (if $>150 \mathrm{mg} / \mathrm{L}=4$ points); ii) total white cell count $\left(<15 \times 10^{6} / \mathrm{mm}^{3}=0\right.$ points; $15-25=1$ points; $>25=2$ points); iii) hemoglobin $(>13.5 \mathrm{~g} / \mathrm{dL}=0$ points; $11-13.5=1$ point; $<11=2$ points $)$; iv) sodium $(<135$ $\mathrm{mmol} / \mathrm{L}=2$ points $) ; \mathrm{v})$ creatinine $(>141 \mu \mathrm{mol} / \mathrm{L}=2$ points); vi) glucose ( $>10 \mu \mathrm{mol} / \mathrm{L}=1$ point).

A score higher than 6 indicates that NF should be considered.

In our case report, the patient had a LRINEC score of 10 .

Nevertheless, the LRINEC score was never validated and the authors noted that other pathological conditions could be responsible to the same laboratory data changes.

The review of Borschitz et al. ${ }^{4}$ suggests a LRINEC modified score, considering fibrinogen levels and erythrocyte count, instead of sodium and glucose serum levels, and some clinical parameters (pain, fever, tachycardia and signs of renal injury). A score $\geq 8$ is for strong suspicion of NF, while $\leq 5$ is no suspicion.

A recent retrospective analysis ${ }^{7}$ studied the role of procalcitonin to identify cases of sepsis in NF patients, showing that initial procalcitonin concentration in NF is a prognostic value of sepsis.

It is also important to consider the clinical history of patients and the clinical presentation in the Emergency Department where the first evaluation is assessed, in order to detect the presence of NF risk factors and to expect a possible evolution to sepsis or multi-organ failure.

In our case report, the patient was affected by diabetes mellitus (DM).

DM is often reported in NF cases, with a mean of $44.5 \%{ }^{5}$ and it is considered as a predisposal disease to NF also because of the high rate of cutaneous infections or ulcers. In the same study it is showed a strong correlation between DM and limb amputation, but not with death.

In a recent retrospective study Cheng et $\mathrm{al} .{ }^{8} \mathrm{col}-$ lected data of 165 NF patients in National Taiwan University Hospital in the period of January 1997 and February 2013. Among these patients, 84 (51\%) had
DM and, compared with the non-diabetic patients, were older and with high rate of serum glucose and potassium at the admission. Moreover, patients affected by DM had a higher rate of limb amputation than the nondiabetic patients. This study also shows that diabetic patients are more susceptible to polymicrobial and monomicrobial Klebsiella pneumonia infections.

Thus, the presence of DM diagnosis with high serum potassium levels and bacteremia at admission represents a high risk factor of mortality in a NF suspect.

In a recent study ${ }^{9}$ authors considered the association between diabetic foot ulcers in DM and NF, leading to lower extremity amputation. Among 2265 diabetic patients analyzed in the period between 2009 and 2014 in Chang Gung Memorial Hospital in Taiwan, $110(4.9 \%)$ had lower extremity NF and DM type 2. In most of the patients with a NF diagnosis $(90.9 \%)$ there were diabetic ulcers.

In conclusion, clinical signs and laboratory data may help physicians to early recognize NF patients and to plan an adequate management in the Emergency Department.

\section{References}

1. De Prost N, Sbidian E, Chosidow O, et al. Management of necrotizing soft tissue infection in the intensive care unit: results of an international survey. Intensive Care Med 2015;41:1506-8.

2. Elliott DC, Kufera JA, Myers RA. Necrotizing soft tissue infections. Risk factors for mortality and strategies for management. Ann Surg 1996;224:672-83.

3. Cheung JPY, Fung B, Tang WM, Ip WY. A review of necrotizing fasciitis iin the extremities. Hong Kong Med J 2009; 15:44-52.

4. Borschitz T, Schlicht S, Siegel E, et al. Improvement of a clinical score for necrotizing fasciitis: "pain out proportion" and high CRP levels aid the diagnosis. PLoS One 2015;10:e0132775.

5. Goh T, Goh LG, Ang CH, et al. Early diagnosis of necrotizing fasciitis. Br J Surg 2014;101:e119-25.

6. Wong CH, Khin LW, Heng KS, et al. The LRINEC (Laboratory Risk Indicator for Necrotizing Fasciitis) score: a tool for distinguishing necrotizing fasciitis from other soft tissue infections. Crit Care Med 2004;32:1535-41.

7. Al-Thani H, El Menyar A, Shaikh N, et al. Risk stratification of necrotizing fasciitis based on the initial procalcitonin concentration: a single center observational study. Surg Infect (Larchmt) 2015;16:806-12.

8. Cheng NC, Tai HC, Chang SC, et al. Necrotizing fasciitis in patients with diabetes mellitus: clinical characteristics and risk factors for mortality. BMC Infect Dis 2015; 15:417.

9. Chen IW, Yang HM, Chiu CH, et al. Clinical characteristics and risk factor analysis for lower-extremity amputations in diabetic patients with foot ulcer complicated by necrotizing fasciitis. Medicine (Baltimore) 2015; 94:e1957. 